\title{
On fractional Hahn calculus
}

\section{Tanapat Brikshavana and Thanin Sitthiwirattham*}

\author{
${ }^{*}$ Correspondence: \\ thanin_sit@dusit.ac.th \\ Mathematics Department, Faculty \\ of Science and Technology, Suan \\ Dusit University, Bangkok, Thailand
}

\begin{abstract}
In this paper, the new concepts of Hahn difference operators are introduced. The properties of fractional Hahn calculus in the sense of a forward Hahn difference operator are introduced and developed.
\end{abstract}

MSC: 39A10; 39A13; 39A70

Keywords: fractional Hahn integral; fractional Hahn difference operator; Jackson-Nörlund integral

\section{Introduction}

The quantum calculus, known as calculus without the consideration of limits, involves sets of non-differentiable functions. There are many types of quantum difference operators such as the Jackson $q$-difference operator, the forward (delta) difference operator, the backward (nabla) difference operator, and so on. These operators are employed in many applications, for example, combinatorics, orthogonal polynomials, basic hypergeometric functions, hypergeometric series, complex analysis, the calculus of variations, the theory of relativity, quantum mechanics, and particle physics [1-9].

In 1949, Hahn [10] introduced the Hahn difference operator $D_{q, \omega}$ as follows:

$$
D_{q, \omega} f(t):=\frac{f(q t+\omega)-f(t)}{t(q-1)+\omega}, \quad t \neq \frac{\omega}{1-q} .
$$

This operator is created with a combination of two well-known operators, the forward difference operator and the Jackson $q$-difference operator. We observe that

$$
\begin{aligned}
& D_{q, \omega} f(t)=\Delta_{\omega} f(t) \quad \text { whenever } q=1, \\
& D_{q, \omega} f(t)=D_{q} f(t) \quad \text { whenever } \omega=0, \quad \text { and } \\
& D_{q, \omega} f(t)=f^{\prime}(t) \quad \text { whenever } q=1, \omega \rightarrow 0 .
\end{aligned}
$$

Particularly, the Hahn difference operator has been employed to construct families of orthogonal polynomials as well as to examine some approximation problems (see [11-13] and the references therein).

In 2009 Aldwoah [14, 15] ( $\mathrm{PhD}$ thesis supervised by Annaby and Hamza) defined the right inverse of $D_{q, \omega}$ in terms of both the Jackson $q$-integral containing the right inverse of $D_{q}[16]$ and the Nörlund sum involving the right inverse of $\Delta_{\omega}[16]$.

(c) The Author(s) 2017. This article is distributed under the terms of the Creative Commons Attribution 4.0 International License (http://creativecommons.org/licenses/by/4.0/), which permits unrestricted use, distribution, and reproduction in any medium, provided you give appropriate credit to the original author(s) and the source, provide a link to the Creative Commons license, and indicate if changes were made. 
Malinowska and Torres [17, 18] studied the Hahn quantum variational calculus in 2010. Moreover, in 2013, Malinowska and Martins [19] studied the generalized transversality conditions for the Hahn quantum variational calculus. In the same year, Hamza et al. [2022] studied the theory of linear Hahn difference equations. They established the existence and uniqueness results for the initial value problems for Hahn difference equations by using the method of successive approximations. In addition, they proved Gronwall's and Bernoulli's inequalities with respect to the Hahn difference operator and investigated the mean value theorems, Leibniz's rule and Fubini's theorem for this calculus. For the boundary value problems, in 2016, Sitthiwirattham [23] considered a nonlinear Hahn difference equation with nonlocal boundary value conditions

$$
\begin{aligned}
& D_{q, \omega}^{2} x(t)+f\left(t, x(t), D_{p, \theta} x(p t+\theta)\right)=0, \quad t \in\left[\omega_{0}, T\right]_{q, \omega}, \\
& x\left(\omega_{0}\right)=\varphi(x), \\
& x(T)=\lambda x(\eta), \quad \eta \in\left(\omega_{0}, T\right)_{q, \omega},
\end{aligned}
$$

where $0<q<1,0<\omega<T, \omega_{0}:=\frac{\omega}{1-q}, 1 \leq \lambda<\frac{T-\omega_{0}}{\eta-\omega_{0}}, p=q^{m}, m \in \mathbb{N}, \theta=\omega\left(\frac{1-p}{1-q}\right), f:$ $\left[\omega_{0}, T\right]_{q, \omega} \times \mathbb{R} \times \mathbb{R} \rightarrow \mathbb{R}$ is a continuous function, and $\varphi: C\left(\left[\omega_{0}, T\right]_{q, \omega}, \mathbb{R}\right) \rightarrow \mathbb{R}$ is a given functional. Moreover, in this year 2017, Sriphanomwan et al. [24] studied a nonlocal boundary value problem for second-order nonlinear Hahn integro-difference equation with integral boundary condition

$$
\begin{aligned}
& D_{q, \omega}^{2} x(t)=f\left(t, x(t), D_{p, \theta} x(p t+\theta), \Psi_{p, \theta} x(p t+\theta)\right), \quad t \in\left[\omega_{0}, T\right]_{q, \omega}, \\
& x\left(\omega_{0}\right)=x(T), \\
& x(\eta)=\mu \int_{\omega_{0}}^{T} g(s) x(s) d_{q, \omega} s, \quad \eta \in\left(\omega_{0}, T\right)_{q, \omega},
\end{aligned}
$$

where $0<q<1,0<\omega<T, \omega_{0}:=\frac{\omega}{1-q}, \mu \int_{\omega_{0}}^{T} g(r) d_{q, \omega} r \neq 1, \mu \in \mathbb{R}, p=q^{m}, m \in \mathbb{N}, \theta=\omega\left(\frac{1-p}{1-q}\right)$, $f \in C\left(\left[\omega_{0}, T\right]_{q, \omega} \times \mathbb{R} \times \mathbb{R} \times \mathbb{R} \rightarrow \mathbb{R}\right)$, and $g \in C\left(\left[\omega_{0}, T\right]_{q, \omega} \times \mathbb{R}^{+}\right)$are given functions, and for $\varphi \in C\left(\left[\omega_{0}, T\right]_{q, \omega} \times\left[\omega_{0}, T\right]_{q, \omega},[0, \infty)\right)$

$$
\Psi_{p, \theta} x(t):=\int_{\omega_{0}}^{t} \varphi(t, p s+\theta) x(p s+\theta) d_{p, \theta} s
$$

In particular, the fractional Hahn difference equations have not been studied. We observe that in 2010, Čermák and Nechvátal [25] introduced the fractional $(q, h)$-difference operator and the fractional $(q, h)$-integral for $q>1$. Čermák et al. [26] studied discrete Mittag-Leffler functions in linear fractional difference equations for $q>1$ in 2011. In the same year Rahmat $[27,28]$ investigated the $(q, h)$-Laplace transform and some $(q, h)$ analogues of integral inequalities on discrete time scales for $q>1$. Recently Du et al. [29] studied the monotonicity and convexity for nabla fractional $(q, h)$-difference for $q>0, q \neq 1$ in 2016. However, these operators are not satisfied with fractional Hahn operators because fractional Hahn operators require the condition $0<q<1$.

The gap mentioned above is the motivation for this research. The aim of this paper is to introduce new concepts of Hahn difference operator, the fractional Hahn integral, the fractional Hahn difference operators of Riemann-Liouville and Caputo types. We organize 
this paper as follows. In Section 2, some basic formulas of the Hahn difference operator and the associated Jackson-Nörlund integral calculus are briefly reviewed. In Section 3, we present the fractional Hahn integral and develop some fundamental properties. The fractional Hahn difference operators are presented in Sections 4 and 5.

\section{Preliminary definitions and properties}

The following notations, definitions, and lemmas will be used in proving the main results. Let $q \in(0,1), \omega>0, \omega_{0}=\frac{\omega}{1-q}$, and define

$$
[n]_{q, \omega}=[n]_{q}:=\frac{1-q^{n}}{1-q}=q^{n-1}+\cdots+q+1 \quad \text { and } \quad[n]_{q, \omega} !=[n]_{q} !:=\prod_{k=1}^{n} \frac{1-q^{k}}{1-q}
$$

The $q$-analogue of the power function $(a-b) \frac{n}{q}$ with $n \in \mathbb{N}_{0}:=[0,1,2, \ldots]$ is

$$
(a-b) \frac{0}{q}:=1, \quad(a-b) \frac{n}{q}:=\prod_{k=0}^{n-1}\left(a-b q^{k}\right), \quad a, b \in \mathbb{R}
$$

The $q, \omega$-analogue of the power function $(a-b) \frac{n}{q, \omega}$ with $n \in \mathbb{N}_{0}:=[0,1,2, \ldots]$ is

$$
(a-b) \frac{0}{q, \omega}:=1, \quad(a-b) \frac{n}{q, \omega}:=\prod_{k=0}^{n-1}\left[a-\left(b q^{k}+\omega[k]_{q}\right)\right], \quad a, b \in \mathbb{R} .
$$

More generally, if $\alpha \in \mathbb{R}$, we have

$$
\begin{aligned}
& (a-b) \frac{\alpha}{q}=a^{\alpha} \prod_{n=0}^{\infty} \frac{1-\left(\frac{b}{a}\right) q^{n}}{1-\left(\frac{b}{a}\right) q^{\alpha+n}}, \quad a \neq 0, \\
& (a-b) \frac{\alpha}{q, \omega}=\left(a-\omega_{0}\right)^{\alpha} \prod_{n=0}^{\infty} \frac{1-\left(\frac{b-\omega_{0}}{a-\omega_{0}}\right) q^{n}}{1-\left(\frac{b-\omega_{0}}{a-\omega_{0}}\right) q^{\alpha+n}}=\left(\left(a-\omega_{0}\right)-\left(b-\omega_{0}\right)\right)_{q}^{\frac{\alpha}{q}} \quad a \neq \omega_{0} .
\end{aligned}
$$

Note that $a_{q}^{\frac{\alpha}{q}}=a^{\alpha}$ and $\left(a-\omega_{0}\right) \frac{\alpha}{q, \omega}=\left(a-\omega_{0}\right)^{\alpha}$. We use the notation $(0) \frac{\alpha}{q}=\left(\omega_{0}\right) \frac{\alpha}{q, \omega}=0$ for $\alpha>0$. For $\alpha, \beta, \gamma, \lambda \in \mathbb{R}$, we have

(A1) $(\lambda \beta-\gamma \lambda)^{\frac{\alpha}{q}}=\gamma^{\alpha}(\beta-\lambda)^{\frac{\alpha}{q}}$,

(A2) $(\beta-\gamma) \frac{\alpha+\gamma}{q, \omega}=(\beta-\gamma) \frac{\alpha}{q, \omega}\left(\beta-q^{\alpha \omega} \lambda\right) \frac{\gamma}{q, \omega}$,

(A3) $(t-s) \frac{\alpha}{q, \omega}=0, t \geq s, \alpha \notin \mathbb{N}_{0}$, for $t, s \in I_{q, \omega}^{T}:=\left\{q^{k} T+\omega[k]_{q}: k \in \mathbb{N}_{0}\right\}$.

The $q$-gamma and $q$-beta functions are defined by

$$
\begin{aligned}
\Gamma_{q}(x): & =\frac{(1-q) \frac{x-1}{q}}{(1-q)^{x-1}}, \quad x \in \mathbb{R} \backslash\{0,-1,-2, \ldots\}, \\
B_{q}(x, s) & :=\int_{0}^{1} t^{x-1}(1-q t) \frac{s-1}{q} d_{q} t \\
& =(1-q) q^{n} \sum_{n=0}^{\infty}\left(q^{n}\right)^{\alpha-1}\left(1-q^{n+1}\right) \frac{\beta-1}{q}=\frac{\Gamma_{q}(x) \Gamma_{q}(s)}{\Gamma_{q}(x+s)},
\end{aligned}
$$

respectively. 
The $q, \omega$-forward jump operator, $q, \omega$-backward jump operator, and $q, \omega$-forward graininess function are defined by

$$
\begin{aligned}
& \sigma_{q, \omega}(t):=q t+\omega, \quad \rho_{q, \omega}(t):=\frac{t-\omega}{q}, \quad \text { and } \\
& \mu_{q, \omega}(t):=t(q-1)+\omega, \quad \text { respectively. }
\end{aligned}
$$

Definition 2.1 For $q \in(0,1), \omega>0$, and $f$ defined on an interval $I_{q, \omega}^{T} \subseteq \mathbb{R}$ containing $\omega_{0}:=$ $\frac{\omega}{1-q}$, the Hahn difference of $f$ is defined by

$$
D_{q, \omega} f(t):=\frac{f\left(\sigma_{q, \omega}(t)\right)-f(t)}{\mu_{q, \omega}(t)} \quad \text { for } t \in I_{q, \omega}^{T}\left\{\omega_{0}\right\}
$$

and $D_{q, \omega} f\left(\omega_{0}\right)=f^{\prime}\left(\omega_{0}\right)$, provided that $f$ is differentiable at $\omega_{0}$. We call $D_{q, \omega} f$ the $q, \omega$ derivative of $f$ and say that $f$ is $q, \omega$-differentiable on $I_{q, \omega}^{T}$.

Lemma 2.1 ([15]) Let $f, g$ be q, $\omega$-differentiable on $I_{q, \omega}^{T}$. The Hahn difference operator has the following properties:

(a) $D_{q, \omega}[f(t)+g(t)]=D_{q, \omega} f(t)+D_{q, \omega} g(t)$;

(b) $D_{q, \omega}[\alpha f(t)]=\alpha D_{q, \omega} f(t)$ for $\alpha \in \mathbb{R}$;

(c) $D_{q, \omega}[f(t) g(t)]=f(t) D_{q, \omega} g(t)+g\left(\sigma_{q, \omega}\right) D_{q, \omega} f(t)$;

(d) $D_{q, \omega}\left[\frac{f(t)}{g(t)}\right]=\frac{g(t) D_{q, \omega} f(t)-f(t) D_{q, \omega} g(t)}{g(t) g\left(\sigma_{q, \omega}\right)}$ for $g(t) g\left(\sigma_{q, \omega}\right) \neq 0$.

Lemma 2.2 Let $t \in I_{q, \omega}^{T}, q \in(0,1), \omega>0$, and $\alpha, \beta \in \mathbb{R}$. Then the following statements are true:

(a) $D_{q, \omega}(t-\beta)_{q, \omega}^{\frac{\alpha}{q}}=[\alpha]_{q}\left(\rho_{q, \omega}(t)-\beta\right)^{\frac{\alpha-1}{q, \omega}}$;

(b) $D_{q, \omega}(\beta-t)^{\frac{\alpha}{q}, \omega}=-[\alpha]_{q}(\beta-t) \frac{\alpha-1}{q, \omega}$.

Proof Let $\hat{t}:=t(1-q)-\omega, \hat{\beta}:=\beta(1-q)-\omega$. Since

$$
D_{q, \omega} f(t)=\frac{f\left(\sigma_{q, \omega}(t)\right)-f(t)}{\mu_{q, \omega}(t)}=\frac{f\left(\rho_{q, \omega}(t)\right)-f(t)}{\left(\frac{1}{q}-1\right) t-\frac{\omega}{q}},
$$

we have

$$
\begin{aligned}
D_{q, \omega}(t-\beta) \frac{\alpha}{q, \omega} & =D_{q, \omega}\left[\left(t-\omega_{0}\right)^{\alpha} \frac{\prod_{k=0}^{\infty}\left(1-\frac{\beta-\omega_{0}}{t-\omega_{0}} q^{k}\right)}{\prod_{k=0}^{\infty}\left(1-\frac{\beta-\omega_{0}}{t-\omega_{0}} q^{k+\alpha}\right)}\right] \\
& =\frac{q}{\hat{t}}\left(\frac{\hat{t}}{1-q}\right)^{\alpha}\left[\frac{\prod_{k=0}^{\infty}\left(1-\frac{\hat{\beta}}{\hat{t}} q^{k+1}\right)}{q^{\alpha} \prod_{k=0}^{\infty}\left(1-\frac{\hat{\beta}}{\hat{t}} q^{k+\alpha+1}\right)}-\frac{\prod_{k=0}^{\infty}\left(1-\frac{\hat{\beta}}{\hat{t}} q^{k}\right)}{\prod_{k=0}^{\infty}\left(1-\frac{\hat{\beta}}{\hat{t}} q^{k+\alpha}\right)}\right] \\
& =q\left(\frac{\hat{t}}{1-q}\right)^{\alpha} \frac{\prod_{k=0}^{\infty}\left(1-\frac{\hat{\beta}}{\hat{t}} q^{k+1}\right)}{\prod_{k=0}^{\infty}\left(1-\frac{\hat{\beta}}{\hat{t}} q^{k+\alpha}\right)}\left[\frac{\left(1-\frac{\hat{\beta}}{\hat{t}} q^{\alpha}\right)-q^{\alpha}\left(1-\frac{\hat{\beta}}{\hat{t}}\right)}{\hat{t}}\right] \\
& =\left(\frac{\hat{t}}{q(1-q)}\right)^{\alpha-1} \frac{\prod_{k=0}^{\infty}\left(1-\frac{\hat{\beta}}{\hat{t}} q^{k+1}\right)}{\prod_{k=0}^{\infty}\left(1-\frac{\hat{\beta}}{\hat{t}} q^{k+\alpha}\right)}\left[\frac{1-q^{\alpha}}{1-q}\right]
\end{aligned}
$$




$$
\begin{aligned}
& =\left(\rho_{q, \omega}(t)-\omega_{0}\right)^{\alpha-1} \frac{\prod_{k=0}^{\infty}\left(1-\frac{\beta-\omega_{0}}{\rho_{q, \omega}(t)-\omega_{0}} q^{k}\right)}{\prod_{k=0}^{\infty}\left(1-\frac{\beta-\omega_{0}}{\rho_{q, \omega}(t)-\omega_{0}} q^{k+\alpha-1}\right)}\left[\frac{1-q^{\alpha}}{1-q}\right] \\
& =[\alpha]_{q}\left(\rho_{q, \omega}(t)-\beta\right)_{q, \omega}^{\alpha-1} .
\end{aligned}
$$

So, (a) holds. Proceeding similarly as above, we find that (b) holds.

Letting $a, b \in I \subseteq \mathbb{R}$ with $a<\omega_{0}<b$ and $[k]_{q}=\frac{1-q^{k}}{1-q}, k \in \mathbb{N}_{0}:=\mathbb{N} \cup\{0\}$, we define the $q, \omega$-interval by

$$
\begin{aligned}
{[a, b]_{q, \omega} } & :=\left\{q^{k} a+\omega[k]_{q}: k \in \mathbb{N}_{0}\right\} \cup\left\{q^{k} b+\omega[k]_{q}: k \in \mathbb{N}_{0}\right\} \cup\left\{\omega_{0}\right\} \\
& =\left[a, \omega_{0}\right]_{q, \omega} \cup\left[\omega_{0}, b\right]_{q, \omega} \\
& =(a, b)_{q, \omega} \cup\{a, b\}=[a, b)_{q, \omega} \cup\{b\}=(a, b]_{q, \omega} \cup\{a\} .
\end{aligned}
$$

Observe that, for each $s \in[a, b]_{q, \omega}$, the sequence $\left\{\sigma_{q, \omega}^{k}(s)\right\}_{k=0}^{\infty}=\left\{q^{k} s+\omega[k]_{q}\right\}_{k=0}^{\infty}$ is uniformly convergent to $\omega_{0}$.

Also we define the forward jump operator $\sigma_{q, \omega}^{k}(t):=q^{k} t+\omega[k]_{q}$ and the backward jump operator $\rho_{q, \omega}^{k}(t):=\frac{t-\omega[k] q}{q^{k}}$ for $k \in \mathbb{N}$.

Definition 2.2 Let $I$ be any closed interval of $\mathbb{R}$ containing $a, b$, and $\omega_{0}$. Assuming that $f: I \rightarrow \mathbb{R}$ is a given function, we define $q, \omega$-integral of $f$ from $a$ to $b$ by

$$
\int_{a}^{b} f(t) d_{q, \omega} t:=\int_{\omega_{0}}^{b} f(t) d_{q, \omega} t-\int_{\omega_{0}}^{a} f(t) d_{q, \omega} t
$$

where

$$
\int_{\omega_{0}}^{x} f(t) d_{q, \omega} t:=[x(1-q)-\omega] \sum_{k=0}^{\infty} q^{k} f\left(x q^{k}+\omega[k]_{q}\right), \quad x \in I
$$

provided that the series converges at $x=a$ and $x=b . f$ is called $q, \omega$-integrable on $[a, b]$, and the sum to the right-hand side of the above equation will be called the Jackson-Nörlund sum.

We note that:

(i) The actual domain of function $f$ is defined on $[a, b]_{q, \omega} \subset I$.

(ii) For each $x \in[a, b]_{q, \omega}$, we have $\lim _{k \rightarrow \infty} \sigma_{q, \omega}^{k}(x)=\omega_{0}$. It implies that

$$
\int_{\omega_{0}}^{x} f(t) d_{q, \omega} t=\sum_{s=x}^{\rho_{q, \omega}\left(\omega_{0}\right)}[s(1-q)-\omega] f(s) .
$$

Lemma 2.3 ([15]) Let $q \in(0,1), \omega>0, a, b \in I_{q, \omega}^{T}$, and $f, g$ be $q$, $\omega$-integrable on $I_{q, \omega}^{T}$. Then the following formulas hold:

(a) $\int_{a}^{a} f(t) d_{q, \omega} t=0$;

(b) $\int_{a}^{b} \alpha f(t) d_{q, \omega} t=\alpha \int_{a}^{b} f(t) d_{q, \omega} t, \alpha \in \mathbb{R}$;

(c) $\int_{a}^{b} f(t) d_{q, \omega} t=-\int_{b}^{a} f(t) d_{q, \omega} t$;

(d) $\int_{a}^{b} f(t) d_{q, \omega} t=\int_{c}^{b} f(t) d_{q, \omega} t+\int_{a}^{c} f(t) d_{q, \omega} t, c \in I_{q, \omega}^{T}, a<c<b$; 
(e) $\int_{a}^{b}[f(t)+g(t)] d_{q, \omega} t=\int_{a}^{b} f(t) d_{q, \omega} t+\int_{a}^{b} g(t) d_{q, \omega} t$;

(f) $\int_{a}^{b}\left[f(t) D_{q, \omega} g(t)\right] d_{q, \omega} t=[f(t) g(t)]_{a}^{b}-\int_{a}^{b}\left[g\left(\sigma_{q, \omega}(t)\right) D_{q, \omega} f(t)\right] d_{q, \omega} t$.

We next introduce the fundamental theorem and Leibniz formula of Hahn calculus.

Lemma 2.4 (Fundamental theorem of Hahn calculus [14]) Let $f: I \rightarrow \mathbb{R}$ be continuous at $\omega_{0}$. Define

$$
F(x):=\int_{\omega_{0}}^{x} f(t) d_{q, \omega} t, \quad x \in I .
$$

Then $F$ is continuous at $\omega_{0}$. Furthermore, $D_{q, \omega} F(x)$ exists for every $x \in I$ and

$$
D_{q, \omega} F(x)=f(x) .
$$

Conversely,

$$
\int_{a}^{b} D_{q, \omega} f(t) d_{q, \omega} t=f(b)-f(a) \text { for all } a, b \in I .
$$

Lemma 2.5 (Leibniz formula of Hahn calculus [22]) Let $f: I_{q, \omega}^{T} \times I_{q, \omega}^{T} \rightarrow \mathbb{R}$. Then

$$
D_{q, \omega}\left[\int_{\omega_{0}}^{t} f(t, s) d_{q, \omega} s\right]=\int_{\omega_{0}}^{t}{ }_{t} D_{q, \omega} f(t, s) d_{q, \omega} s+f\left(\sigma_{q, \omega}(t), t\right),
$$

where ${ }_{t} D_{q, \omega}$ is Hahn difference with respect to $t$.

Next, we give some auxiliary lemmas used for simplifying calculations.

Lemma 2.6 ([23]) Let $q \in(0,1), \omega>0$ and $f: I \rightarrow \mathbb{R}$ be continuous at $\omega_{0}$. Then

$$
\int_{\omega_{0}}^{t} \int_{\omega_{0}}^{r} f(s) d_{q, \omega} s d_{q, \omega} r=\int_{\omega_{0}}^{t} \int_{q s+\omega}^{t} f(s) d_{q, \omega} r d_{q, \omega} s .
$$

Lemma 2.7 ([23]) Let $q \in(0,1)$ and $\omega>0$. Then

$$
\int_{\omega_{0}}^{t} d_{q, \omega} s=t-\omega_{0} \text { and } \int_{\omega_{0}}^{t}[t-(q s+\omega)] d_{q, \omega} s=\frac{\left(t-\omega_{0}\right)^{2}}{1+q} .
$$

\section{Fractional Hahn integral}

Now, we introduce fractional Hahn integral.

Definition 3.1 For $\alpha, \omega>0, q \in(0,1)$ and $f$ defined on $\left[\omega_{0}, T\right]_{q, \omega}$, the fractional Hahn integral is defined by

$$
\begin{aligned}
\mathcal{I}_{q, \omega}^{\alpha} f(t) & :=\frac{1}{\Gamma_{q}(\alpha)} \int_{\omega_{0}}^{t}\left(t-\sigma_{q, \omega}(s)\right)_{q, \omega}^{\alpha-1} f(s) d_{q, \omega} s \\
& =\frac{[t(1-q)-\omega]}{\Gamma_{q}(\alpha)} \sum_{n=0}^{\infty} q^{n}\left(t-\sigma_{q, \omega}^{n+1}(t)\right)_{q, \omega}^{\alpha-1} f\left(\sigma_{q, \omega}^{n}(t)\right),
\end{aligned}
$$

and $\left(\mathcal{I}_{q, \omega}^{0} f\right)(t)=f(t)$. 
Since $(1-q) t-\omega=(1-q)\left(t-\omega_{0}\right)$, associated with (A1), we have

$$
\begin{aligned}
\left(t-\sigma_{q, \omega}^{n+1}(t)\right)_{q, \omega}^{\frac{\alpha-1}{2}} & =\left(\left(t-\omega_{0}\right)-\left(\sigma_{q, \omega}^{n+1}(t)-\omega_{0}\right)\right)_{\frac{\alpha-1}{q}} \\
& =\left(\left(t-\omega_{0}\right)-q^{n+1}\left(t-\omega_{0}\right)\right)^{\frac{\alpha-1}{q}} \\
& =\left(t-\omega_{0}\right)^{\alpha-1}\left(1-q^{n+1}\right)_{q}^{\frac{\alpha-1}{q}}
\end{aligned}
$$

It implies that

$$
\mathcal{I}_{q, \omega}^{\alpha} f(t)=\frac{(1-q)\left(t-\omega_{0}\right)^{\alpha}}{\Gamma_{q}(\alpha)} \sum_{n=0}^{\infty} q^{n}\left(1-q^{n+1}\right)_{q}^{\frac{\alpha-1}{q}} f\left(\sigma_{q, \omega}^{n}(t)\right)
$$

Next, we provide some auxiliary lemmas for simplifying calculations.

Lemma 3.1 Let $\alpha, \beta>0, p, q \in(0,1)$, and $\omega>0$. Then

$$
\begin{aligned}
& \int_{\omega_{0}}^{t}\left(t-\sigma_{q, \omega}(s)\right)_{q, \omega}^{\frac{\alpha-1}{}}\left(s-\omega_{0}\right) \frac{\beta}{q, \omega} d_{q, \omega} s=\left(t-\omega_{0}\right)^{\alpha+\beta} B_{q}(\beta+1, \alpha), \\
& \int_{\omega_{0}}^{t} \int_{\omega_{0}}^{x}\left(t-\sigma_{p, \omega}(x)\right) \frac{\alpha-1}{p, \omega}\left(x-\sigma_{q, \omega}(s)\right) \frac{\beta-1}{q, \omega} d_{q, \omega} s d_{p, \omega} x=\frac{\left(t-\omega_{0}\right)^{\alpha+\beta}}{[\beta]_{q}} B_{p}(\beta+1, \alpha) .
\end{aligned}
$$

Proof From the definition of $q, \omega$-analogue of the power function and Definition 3.1, we obtain

$$
\begin{aligned}
& \int_{\omega_{0}}^{t}\left(t-\sigma_{q, \omega}(s)\right)_{q, \omega}^{\frac{\alpha-1}{q}}\left(s-\omega_{0}\right) \frac{\beta}{q, \omega} d_{q, \omega} s \\
& =\left(t-\omega_{0}\right)^{\alpha}(1-q) \sum_{n=0}^{\infty} q^{n}\left(1-q^{n+1}\right)_{q}^{\frac{\alpha-1}{q}}\left(q^{n}\left(t-\omega_{0}\right)\right)^{\beta} \\
& =\left(t-\omega_{0}\right)^{\alpha+\beta}(1-q) \sum_{n=0}^{\infty} q^{n}\left(1-q^{n+1}\right)_{q}^{\frac{\alpha-1}{q}}\left(q^{n}\right)^{\beta} \\
& =\left(t-\omega_{0}\right)^{\alpha+\beta} B_{q}(\beta+1, \alpha),
\end{aligned}
$$

and

$$
\begin{aligned}
& \int_{\omega_{0}}^{t} \int_{\omega_{0}}^{x}\left(t-\sigma_{p, \omega}(x)\right)_{p, \omega}^{\frac{\alpha-1}{}}\left(x-\sigma_{q, \omega}(s)\right) \frac{\beta-1}{q, \omega} d_{q, \omega} s d_{p, \omega} x \\
& =\int_{\omega_{0}}^{t}\left(t-\sigma_{p, \omega}(x) \frac{\alpha-1}{p, \omega}\left[\int_{\omega_{0}}^{x}\left(x-\sigma_{q, \omega}(s)\right) \frac{\beta-1}{q, \omega} d_{q, \omega} s\right] d_{p, \omega} x\right. \\
& =\frac{1}{[\beta]_{q}} \int_{\omega_{0}}^{t}\left(t-\sigma_{p, \omega}(x) \frac{\alpha-1}{p, \omega}\left(x-\omega_{0}\right)^{\beta} d_{p, \omega} x\right. \\
& =\frac{\left(t-\omega_{0}\right)^{\alpha+\beta}}{[\beta]_{q}} B_{p}(\beta+1, \alpha) .
\end{aligned}
$$

In the next theorems we introduce the properties of fractional Hahn integral as the following theorem. 
Brikshavana and Sitthiwirattham Advances in Difference Equations ( 2017) 2017:354

Page 8 of 15

Theorem 3.1 For $f: I_{q, \omega}^{T} \rightarrow \mathbb{R}, \alpha>0, q \in(0,1), \omega>0$, and $a \in I_{q, \omega}^{T}$,

$$
\mathcal{I}_{q, \omega}^{\alpha} f(t)=\mathcal{I}_{q, \omega}^{\alpha+1}\left[D_{q, \omega} f(t)\right]+\frac{f\left(\omega_{0}\right)}{\Gamma_{q}(\alpha+1)}\left(t-\omega_{0}\right)^{\alpha}
$$

Proof Using Lemma 2.2(b) and Lemma 2.4, we obtain

$$
\begin{aligned}
\mathcal{I}_{q, \omega}^{\alpha} f(t) & =-\frac{1}{[\alpha]_{q} \Gamma_{q}(\alpha)} \int_{a}^{t} D_{q, \omega}(t-s)_{q, \omega}^{\alpha} f(s) d_{q, \omega} s \\
& =\frac{1}{\Gamma_{q}(\alpha+1)}\left[\left(t-\omega_{0}\right)_{\frac{\alpha}{q}, \omega}^{\alpha} f\left(\omega_{0}\right)+\int_{a}^{t}\left(t-\sigma_{q, \omega}\right) \frac{\alpha}{q, \omega} D_{q, \omega} f(s) d_{q, \omega} s\right] \\
& =\mathcal{I}_{q, \omega}^{\alpha+1}\left[D_{q, \omega} f(t)\right]+\frac{f\left(\omega_{0}\right)}{\Gamma_{q}(\alpha+1)}\left(t-\omega_{0}\right)_{q, \omega}^{\frac{\alpha}{q}} \\
& =\mathcal{I}_{q, \omega}^{\alpha+1}\left[D_{q, \omega} f(t)\right]+\frac{f\left(\omega_{0}\right)}{\Gamma_{q}(\alpha+1)}\left(t-\omega_{0}\right)^{\alpha} .
\end{aligned}
$$

Theorem 3.2 For $f: I_{q, \omega}^{T} \rightarrow \mathbb{R}, \alpha, \beta>0, q \in(0,1), \omega>0$, and $a \in I_{q, \omega}^{T}$,

$$
\int_{\omega_{0}}^{a}\left(t-\sigma_{q, \omega}(s)\right) \frac{\beta-1}{q, \omega} \mathcal{I}_{q, \omega}^{\alpha} f(t) d_{q, \omega} s=0 .
$$

Proof For $n \in \mathbb{N}_{0}$,

$$
\begin{aligned}
\mathcal{I}_{q, \omega}^{\alpha} f\left(\sigma_{q, \omega}^{n}(a)\right) & =\frac{1}{\Gamma_{q}(\alpha)} \int_{\omega_{0}}^{\sigma_{q, \omega}^{n}(a)}\left(\sigma_{q, \omega}^{n}(a)-\sigma_{q, \omega}(s)\right)_{q, \omega}^{\alpha-1} f(s) d_{q, \omega} s \\
& =\frac{\left[\sigma_{q, \omega}^{n}(a)(1-q)-\omega\right]}{\Gamma_{q}(\alpha)} \sum_{k=0}^{\infty} q^{k}\left(\sigma_{q, \omega}^{n}(a)-\sigma_{q, \omega}^{n+k}(a)\right)_{q, \omega}^{\frac{\alpha-1}{} f\left(\sigma_{q, \omega}^{n+k}(a)\right) .}
\end{aligned}
$$

Employing (A3) implies that $\left(\sigma_{q, \omega}^{n}(a)-\sigma_{q, \omega}^{n+k}(a)\right)_{q, \omega}^{\frac{\alpha-1}{q}}=0$. Thus,

$$
\mathcal{I}_{q, \omega}^{\alpha} f\left(\sigma_{q, \omega}^{n}(a)\right)=0
$$

Finally, using Definition 3.1, we have

$$
\begin{aligned}
& \int_{\omega_{0}}^{a}\left(t-\sigma_{q, \omega}(s)\right) \frac{\beta-1}{q, \omega} \mathcal{I}_{q, \omega}^{\alpha} f(t) d_{q, \omega} s \\
& \quad=[(1-q) a-\omega] \sum_{k=0}^{\infty} q^{k}\left(t-\sigma_{q, \omega}^{k+1}(a)\right) \frac{\beta-1}{q, \omega}\left[\mathcal{I}_{q, \omega}^{\alpha} f\left(\sigma_{q, \omega}^{k}(a)\right)\right]=0 .
\end{aligned}
$$

Theorem 3.3 For $f: I_{q, \omega}^{T} \rightarrow \mathbb{R}, \alpha, \beta>0, q \in(0,1), \omega>0$, and $a \in I_{q, \omega}^{T}$,

$$
\mathcal{I}_{q, \omega}^{\alpha}\left[\mathcal{I}_{q, \omega}^{\beta} f(t)\right]=\mathcal{I}_{q, \omega}^{\beta}\left[\mathcal{I}_{q, \omega}^{\alpha} f(t)\right]=\mathcal{I}_{q, \omega}^{\alpha+\beta} f(t)
$$


Brikshavana and Sitthiwirattham Advances in Difference Equations ( 2017) 2017:354

Page 9 of 15

Proof For $t \in I_{q, \omega}^{T}$,

$$
\begin{aligned}
\mathcal{I}_{q, \omega}^{\alpha} \mathcal{I}_{q, \omega}^{\beta} f(t)= & \frac{1}{\Gamma_{q}(\alpha) \Gamma_{q}(\beta)} \int_{\omega_{0}}^{t}\left(t-\sigma_{q, \omega}(s)\right) \frac{\alpha-1}{q, \omega} \mathcal{I}_{q, \omega}^{\beta} f(s) d_{q, \omega} s \\
= & \frac{1}{\Gamma_{q}(\alpha) \Gamma_{q}(\beta)} \int_{\omega_{0}}^{t} \int_{\omega_{0}}^{s}\left(t-\sigma_{q, \omega}(s)\right)_{q, \omega}^{\frac{\alpha-1}{}}\left(s-\sigma_{q, \omega}(x)\right) \frac{\beta-1}{q, \omega} f(x) d_{q, \omega} x d_{q, \omega} s \\
= & \frac{1}{\Gamma_{q}(\alpha) \Gamma_{q}(\beta)} \int_{\omega_{0}}^{t}\left[\int_{\sigma_{q, \omega}(x)}^{t}\left(t-\sigma_{q, \omega}(s)\right)_{q, \omega}^{\alpha-1}\left(s-\sigma_{q, \omega}(x)\right) \frac{\beta-1}{q, \omega} d_{q, \omega} s\right] f(x) d_{q, \omega} x \\
= & \frac{1}{\Gamma_{q}(\alpha) \Gamma_{q}(\beta)}\left\{\int_{\omega_{0}}^{t} \int_{\omega_{0}}^{t}\left(t-\sigma_{q, \omega}(s)\right)_{q, \omega}^{\frac{\alpha-1}{2}}\left(s-\sigma_{q, \omega}(x)\right) \frac{\beta-1}{q, \omega} f(x) d_{q, \omega} s d_{q, \omega} x\right. \\
& \left.-\int_{\omega_{0}}^{t} \int_{\omega_{0}}^{\sigma_{q, \omega}(x)}\left(t-\sigma_{q, \omega}(s)\right)_{q, \omega}^{\frac{\alpha-1}{}}\left(s-\sigma_{q, \omega}(x)\right)_{q, \omega}^{\beta-1} f(x) d_{q, \omega} s d_{q, \omega} x\right\} .
\end{aligned}
$$

From Theorem 3.2, we have

$$
\int_{\omega_{0}}^{t} \int_{\omega_{0}}^{\sigma_{q, \omega}(x)}\left(t-\sigma_{q, \omega}(s)\right)_{q, \omega}^{\alpha-1}\left(s-\sigma_{q, \omega}(x)\right) \frac{\beta-1}{q, \omega} f(x) d_{q, \omega} s d_{q, \omega} x=0 .
$$

Therefore,

$$
\mathcal{I}_{q, \omega}^{\alpha} \mathcal{I}_{q, \omega}^{\beta} f(t)=\frac{1}{\Gamma_{q}(\alpha) \Gamma_{q}(\beta)} \int_{\omega_{0}}^{t} \int_{\omega_{0}}^{t}\left(t-\sigma_{q, \omega}(s)\right)_{q, \omega}^{\alpha-1}\left(s-\sigma_{q, \omega}(x)\right) \frac{\beta-1}{q, \omega} f(x) d_{q, \omega} s d_{q, \omega} x .
$$

Similarly, we have $\mathcal{I}_{q, \omega}^{\alpha} \mathcal{I}_{q, \omega}^{\beta} f(t)=\mathcal{I}_{q, \omega}^{\beta} \mathcal{I}_{q, \omega}^{\alpha} f(t)$.

Next, we show that $\mathcal{I}_{q, \omega}^{\alpha} \mathcal{I}_{q, \omega}^{\beta} f(t)=\mathcal{I}_{q, \omega}^{\alpha+\beta} f(t)$,

$$
\begin{aligned}
\mathcal{I}_{q, \omega}^{\alpha} \mathcal{I}_{q, \omega}^{\beta} f(t) & =\frac{1}{\Gamma_{q}(\alpha) \Gamma_{q}(\beta)} \int_{\omega_{0}}^{t}\left[\int_{\sigma_{q, \omega}(x)}^{t}\left(t-\sigma_{q, \omega}(s)\right) \frac{\alpha-1}{q, \omega}\left(s-\sigma_{q, \omega}(x)\right) \frac{\beta-1}{q, \omega} d_{q, \omega} s\right] f(x) d_{q, \omega} x \\
& =\frac{1}{\Gamma_{q}(\alpha) \Gamma_{q}(\beta)} \int_{\omega_{0}}^{t}\left[\mathcal{I}_{q, \omega}^{\alpha}\left(t-\sigma_{q, \omega}(x)\right) \frac{\beta-1}{q, \omega}\right] f(x) d_{q, \omega} x \\
& =\frac{1}{\Gamma_{q}(\beta)} \int_{\omega_{0}}^{t} \frac{\Gamma_{q}(\beta)}{\Gamma_{q}(\alpha+\beta)}\left(t-\sigma_{q, \omega}(x)\right) \frac{\alpha+\beta-1}{q, \omega} f(x) d_{q, \omega} x=\mathcal{I}_{q, \omega}^{\alpha+\beta} f(t)
\end{aligned}
$$

Theorem 3.4 For $: I_{q, \omega}^{T} \rightarrow \mathbb{R}, \alpha, \omega>0$, and $q \in(0,1)$,

$$
\mathcal{I}_{q, \omega}^{\alpha}\left[D_{q, \omega} f(t)\right]=D_{q, \omega}\left[\mathcal{I}_{q, \omega}^{\alpha} f(t)\right]-\frac{\left(t-\omega_{0}\right)^{\alpha-1}}{\Gamma_{q}(\alpha)} f\left(\omega_{0}\right)
$$

Proof Using Lemma 2.1(c) and Lemma 2.2(b), we have

$$
\left(t-\sigma_{q, \omega}(s)\right) \frac{\alpha-1}{q, \omega} D_{q, \omega} f(s)=[\alpha-1]_{q}\left(t-\sigma_{q, \omega}(s)\right) \frac{\alpha-2}{q, \omega} f(s)+{ }_{s} D_{q, \omega}\left[(t-s) \frac{\alpha-1}{q, \omega} f(s)\right] .
$$

So, we obtain

$$
\begin{aligned}
\mathcal{I}_{q, \omega}^{\alpha} D_{q, \omega} f(t) & =\frac{1}{\Gamma_{q}(\alpha)} \int_{\omega_{0}}^{t}\left(t-\sigma_{q, \omega}(s)\right)_{q, \omega}^{\frac{\alpha-1}{2}}\left[D_{q, \omega} f(s)\right] d_{q, \omega} s \\
& =\frac{[\alpha-1]_{q}}{\Gamma_{q}(\alpha)} \int_{\omega_{0}}^{t}\left(t-\sigma_{q, \omega}(s)\right)_{q, \omega}^{\alpha-2} f(s) d_{q, \omega} s+\frac{1}{\Gamma_{q}(\alpha)}\left[(t-s) \frac{\alpha-1}{q, \omega} f(s)\right]_{s=\omega_{0}}^{s=t}
\end{aligned}
$$




$$
\begin{aligned}
& =D_{q, \omega}\left[\mathcal{I}_{q, \omega}^{\alpha} f(t)\right]-\frac{\left(t-\omega_{0}\right)_{\frac{\alpha-1}{q \omega}}}{\Gamma_{q}(\alpha)} f\left(\omega_{0}\right) \\
& =D_{q, \omega}\left[\mathcal{I}_{q, \omega}^{\alpha} f(t)\right]-\frac{\left(t-\omega_{0}\right)^{\alpha-1}}{\Gamma_{q}(\alpha)} f\left(\omega_{0}\right) .
\end{aligned}
$$

Theorem 3.5 For $f: I_{q, \omega}^{T} \rightarrow \mathbb{R}, \alpha, \omega>0, q \in(0,1)$, and $p \in \mathbb{N}$,

$$
\mathcal{I}_{q, \omega}^{\alpha}\left[D_{q, \omega}^{p} f(t)\right]=D_{q, \omega}^{p}\left[\mathcal{I}_{q, \omega}^{\alpha} f(t)\right]-\sum_{k=0}^{p-1} \frac{\left(t-\omega_{0}\right)^{\alpha-p+k}}{\Gamma_{q}(\alpha-p+k+1)}\left[D_{q, \omega}^{k} f\left(\omega_{0}\right)\right] .
$$

Proof Substituting $f$ as $D_{q, \omega} f$ into Theorem 3.4, we have

$$
\begin{aligned}
\mathcal{I}_{q, \omega}^{\alpha}\left[D_{q, \omega}^{2} f(t)\right]= & D_{q, \omega}\left[\mathcal{I}_{q, \omega}^{\alpha} D_{q, \omega} f(t)\right]-\frac{\left(t-\omega_{0}\right)^{\alpha-1}}{\Gamma_{q}(\alpha)}\left[D_{q, \omega} f\left(\omega_{0}\right)\right] \\
= & D_{q, \omega}\left[D_{q, \omega}\left[\mathcal{I}_{q, \omega}^{\alpha} f(t)\right]-\frac{\left(t-\omega_{0}\right)^{\alpha-1}}{\Gamma_{q}(\alpha)} f\left(\omega_{0}\right)\right] \\
& -\frac{\left(t-\omega_{0}\right)^{\alpha-1}}{\Gamma_{q}(\alpha)}\left[D_{q, \omega} f\left(\omega_{0}\right)\right] \\
= & D_{q, \omega}^{2}\left[\mathcal{I}_{q, \omega}^{\alpha} f(t)\right]-\sum_{k=0}^{1} \frac{\left(t-\omega_{0}\right)^{\alpha-2+k}}{\Gamma_{q}(\alpha+k-1)}\left[D_{q, \omega}^{k} f\left(\omega_{0}\right)\right] .
\end{aligned}
$$

Repeating the same procedure as above $p-1$ times, we obtain

$$
\mathcal{I}_{q, \omega}^{\alpha}\left[D_{q, \omega}^{p} f(t)\right]=D_{q, \omega}^{p}\left[\mathcal{I}_{q, \omega}^{\alpha} f(t)\right]-\sum_{k=0}^{p-1} \frac{\left(t-\omega_{0}\right)^{\alpha-p+k}}{\Gamma_{q}(\alpha-p+k+1)}\left[D_{q, \omega}^{k} f\left(\omega_{0}\right)\right] .
$$

\section{Fractional Hahn difference operator of Riemann-Liouville type}

In this section, we introduce a fractional Hahn difference operator of Riemann-Liouville type.

Definition 4.1 For $\alpha, \omega>0, q \in(0,1)$, and $f$ defined on $\left[\omega_{0}, T\right]_{q, \omega}$, the fractional Hahn difference operator of Riemann-Liouville type of order $\alpha$ is defined by

$$
\begin{aligned}
D_{q, \omega}^{\alpha} f(t) & :=\left(D_{q, \omega}^{N} \mathcal{I}_{q, \omega}^{N-\alpha} f\right)(t) \\
& =\frac{1}{\Gamma_{q}(-\alpha)} \int_{\omega_{0}}^{t}\left(t-\sigma_{q, \omega}(s)\right)_{q, \omega}^{\frac{-\alpha-1}{f}} f(s) d_{q, \omega} s,
\end{aligned}
$$

and $D_{q,(\omega)}^{0} f(t)=f(t)$, where $N-1<\alpha<N, N \in \mathbb{N}$.

Theorem 4.1 For $\alpha>0, q \in(0,1), \omega>0$, and $f: I_{q, \omega}^{T} \rightarrow \mathbb{R}$,

$$
\begin{aligned}
D_{q, \omega}^{\alpha} f(t) & =\frac{[(1-q) t-\omega]}{\Gamma_{q}(-\alpha)} \sum_{k=0}^{\infty} q^{k}\left(t-\sigma_{q, \omega}^{k+1}(t)\right)_{q, \omega}^{-\alpha-1} f\left(\sigma_{q, \omega}^{k}(t)\right) \\
& =\frac{(1-q)\left(t-\omega_{0}\right)^{-\alpha}}{\Gamma_{q}(-\alpha)} \sum_{k=0}^{\infty} q^{k}\left(1-q^{k+1}\right)_{q, \omega}^{-\frac{-\alpha-1}{f}} f\left(\sigma_{q, \omega}^{k}(t)\right) .
\end{aligned}
$$


Brikshavana and Sitthiwirattham Advances in Difference Equations ( 2017) 2017:354

Page 11 of 15

Proof For some $N-1<\alpha<N, N \in \mathbb{N}$, by using Definition 4.1 and Lemma 2.5, we have

$$
\begin{aligned}
D_{q, \omega}^{\alpha} f(t)= & D_{q, \omega}^{N} \mathcal{I}_{q, \omega}^{N-\alpha} f(t)=D_{q, \omega}^{N-1} D_{q, \omega} \mathcal{I}_{q, \omega}^{N-\alpha} f(t) \\
= & D_{q, \omega}^{N-1}\left\{D_{q, \omega}\left[\frac{1}{\Gamma_{q}(N-\alpha)} \int_{\omega_{0}}^{t}\left(t-\sigma_{q, \omega}(s)\right)_{q, \omega}^{\frac{N-\alpha-1}{2}} f(s) d_{q, \omega} s\right]\right\} \\
= & D_{q, \omega}^{N-1}\left\{\frac{1}{\Gamma_{q}(N-\alpha-1)} \int_{\omega_{0}}^{t}\left(t-\sigma_{q, \omega}(s)\right)_{q, \omega}^{N-\alpha-2} f(s) d_{q, \omega} s\right. \\
& \left.+\frac{1}{\Gamma_{q}(N-\alpha)}\left(\sigma_{q, \omega}(t)-\sigma_{q, \omega}(t)\right)_{q, \omega}^{N-\alpha-1} f(t)\right\} \\
= & D_{q, \omega}^{N-1}\left\{\frac{1}{\Gamma_{q}(N-\alpha-1)} \int_{\omega_{0}}^{t}\left(t-\sigma_{q, \omega}(s)\right)_{q, \omega}^{\frac{N-\alpha-2}{q}} f(s) d_{q, \omega} s\right\} .
\end{aligned}
$$

Repeating $N-1$ times, we obtain

$$
\begin{aligned}
D_{q, \omega}^{\alpha} f(t)= & D_{q, \omega}^{N-N}\left\{\frac{1}{\Gamma_{q}(-\alpha)} \int_{\omega_{0}}^{t}\left(t-\sigma_{q, \omega}(s)\right)_{q, \omega}^{\frac{-\alpha-1}{2}} f(s) d_{q, \omega} s\right. \\
& \left.+\frac{1}{\Gamma_{q}(1-\alpha)}(-\alpha-1) \frac{-\alpha}{q, \omega} f(t+\alpha+1)\right\} \\
= & \frac{1}{\Gamma_{q}(-\alpha)} \int_{\omega_{0}}^{t}\left(t-\sigma_{q, \omega}(s)\right)_{q, \omega}^{\frac{-\alpha-1}{q}} f(s) d_{q, \omega} s \\
= & \frac{[(1-q) t-\omega]}{\Gamma_{q}(-\alpha)} \sum_{k=0}^{\infty} q^{k}\left(t-\sigma_{q, \omega}^{k+1}(t)\right)_{q, \omega}^{\frac{-\alpha-1}{q}} f\left(\sigma_{q, \omega}^{k}(t)\right) \\
= & \frac{(1-q)\left(t-\omega_{0}\right)^{-\alpha}}{\Gamma_{q}(-\alpha)} \sum_{k=0}^{\infty} q^{k}\left(1-q^{k+1}\right)_{q, \omega}^{\frac{-\alpha-1}{q}} f\left(\sigma_{q, \omega}^{k}(t)\right) .
\end{aligned}
$$

In the following theorem, we introduce the properties of fractional Hahn difference opaerator of Riemann-Liouville type.

Theorem 4.2 For $\alpha>0, q \in(0,1), \omega>0$, and $f: I_{q, \omega}^{T} \rightarrow \mathbb{R}$,

$$
D_{q, \omega}^{\alpha} \mathcal{I}_{q, \omega}^{\alpha} f(t)=f(t)
$$

Proof For some $N-1<\alpha<N, N \in \mathbb{N}$,

$$
\begin{aligned}
D_{q, \omega}^{\alpha} \mathcal{I}_{q, \omega}^{\alpha} f(t) & =D_{q, \omega}^{N} \mathcal{I}_{q, \omega}^{N-\alpha} \mathcal{I}_{q, \omega}^{\alpha} f(t) \\
& =\left[D_{q, \omega}^{N} \mathcal{I}_{q, \omega}^{\alpha}\right]\left(\mathcal{I}_{q, \omega}^{N-\alpha} f(t)\right)=D_{q, \omega}^{N} \mathcal{I}_{q, \omega}^{N} f(t)=f(t) .
\end{aligned}
$$

Theorem 4.3 For $\alpha>0, q \in(0,1), \omega>0$, and $f: I_{q, \omega}^{T} \rightarrow \mathbb{R}$,

$$
\mathcal{I}_{q, \omega}^{\alpha} D_{q, \omega}^{\alpha} f(t)=f(t)-\sum_{k=0}^{N-1} \frac{\left(t-\omega_{0}\right)^{\alpha-N+k}}{\Gamma_{q}(\alpha-N+k+1)}\left[D_{q, \omega}^{\alpha-N+k} f\left(\omega_{0}\right)\right]
$$

where $N-1<\alpha<N, N \in \mathbb{N}$. 
Proof From Definition 4.1, we have

$$
\mathcal{I}_{q, \omega}^{\alpha} D_{q, \omega}^{\alpha} f(t)=\mathcal{I}_{q, \omega}^{\alpha}\left[D_{q, \omega}^{N} \mathcal{I}_{q, \omega}^{N-\alpha} f(t)\right]=\mathcal{I}_{q, \omega}^{\alpha} D_{q, \omega}^{N}\left[\mathcal{I}_{q, \omega}^{N-\alpha} f(t)\right]
$$

Using Theorem 3.5, we obtain

$$
\begin{aligned}
\mathcal{I}_{q, \omega}^{\alpha} D_{q, \omega}^{\alpha} f(t) & =D_{q, \omega}^{N}\left[\mathcal{I}_{q, \omega}^{\alpha} \mathcal{I}_{q, \omega}^{N-\alpha} f(t)\right]-\sum_{k=0}^{N-1} \frac{\left(t-\omega_{0}\right)^{\alpha-N+k}}{\Gamma_{q}(\alpha-N+k+1)} D_{q, \omega}^{k}\left[\mathcal{I}_{q, \omega}^{N-\alpha} f\left(\omega_{0}\right)\right] \\
& =D_{q, \omega}^{N}\left[\mathcal{I}_{q, \omega}^{N} f(t)\right]-\sum_{k=0}^{N-1} \frac{\left(t-\omega_{0}\right)^{\alpha-N+k}}{\Gamma_{q}(\alpha-N+k+1)}\left[D_{q, \omega}^{k-(N-\alpha)} f\left(\omega_{0}\right)\right] \\
& =f(t)-\sum_{k=0}^{N-1} \frac{\left(t-\omega_{0}\right)^{\alpha-N+k}}{\Gamma_{q}(\alpha-N+k+1)}\left[D_{q, \omega}^{\alpha-N+k} f\left(\omega_{0}\right)\right] .
\end{aligned}
$$

Corollary 4.1 Let $\alpha>0, q \in(0,1), \omega>0$, and $f: I_{q, \omega}^{T} \rightarrow \mathbb{R}$,

$$
\mathcal{I}_{q, \omega}^{\alpha} D_{q, \omega}^{\alpha} f(t)=f(t)+C_{1}\left(t-\omega_{0}\right)^{\alpha-1}+\cdots+C_{N}\left(t-\omega_{0}\right)^{\alpha-N}
$$

for some $C_{i} \in \mathbb{R}, i=\mathbb{N}_{1, N}$ and $N-1<\alpha<N, N \in \mathbb{N}$.

\section{Fractional Hahn difference operator of Caputo type}

Now, we introduce a fractional Hahn difference operator of Caputo type.

Definition 5.1 For $\alpha, \omega>0, q \in(0,1)$, and $f$ defined on $\left[\omega_{0}, T\right]_{q, \omega}$, the fractional Hahn difference operator of Caputo type of order $\alpha$ is defined by

$$
\begin{aligned}
{ }^{C} D_{q, \omega}^{\alpha} f(t): & =\left(\mathcal{I}_{q, \omega}^{N-\alpha} D_{q, \omega}^{N} f\right)(t) \\
& =\frac{1}{\Gamma_{q}(N-\alpha)} \int_{\omega_{0}}^{t}\left(t-\sigma_{q, \omega}(s)\right)_{q, \omega}^{\frac{N-\alpha-1}{2} D_{q, \omega}^{N} f(s) d_{q, \omega} s,}
\end{aligned}
$$

and ${ }^{\mathrm{C}} D_{q, \omega}^{0} f(t)=f(t)$, where $N-1<\alpha<N, N \in \mathbb{N}$.

Theorem 5.1 For $\alpha>0, q \in(0,1), \omega>0$, and $f: I_{q, \omega}^{T} \rightarrow \mathbb{R}$

$$
\begin{aligned}
{ }^{\mathrm{C}} D_{q, \omega}^{\alpha} f(t) & =\frac{[(1-q) t-\omega]}{\Gamma_{q}(N-\alpha)} \sum_{k=0}^{\infty} q^{k}\left(t-\sigma_{q, \omega}^{k+1}(t)\right)_{q, \omega}^{N-\alpha-1} D_{q, \omega}^{N} f\left(\sigma_{q, \omega}^{k}(t)\right) \\
& =\frac{(1-q)\left(t-\omega_{0}\right)^{N-\alpha}}{\Gamma_{q}(N-\alpha)} \sum_{k=0}^{\infty} q^{k}\left(1-q^{k+1}\right)_{q, \omega}^{N-\alpha-1} D_{q, \omega}^{N} f\left(\sigma_{q, \omega}^{k}(t)\right),
\end{aligned}
$$

where $N-1<\alpha<N, N \in \mathbb{N}$. 
Proof For $t \in I_{q, \omega}^{T}$, by Definition 5.1, we have

$$
\begin{aligned}
{ }^{\mathrm{C}} D_{q, \omega}^{\alpha} f(t) & =\mathcal{I}_{q, \omega}^{N-\alpha} D_{q, \omega}^{N} f(t)=\frac{1}{\Gamma_{q}(N-\alpha)} \int_{\omega_{0}}^{t}\left(t-\sigma_{q, \omega}(s)\right)_{q, \omega}^{N-\alpha-1} D_{q, \omega}^{N} f(s) d_{q, \omega} s \\
& =\frac{[(1-q) t-\omega]}{\Gamma_{q}(N-\alpha)} \sum_{k=0}^{\infty} q^{k}\left(t-\sigma_{q, \omega}^{k+1}(t)\right)_{q, \omega}^{N-\alpha-1} D_{q, \omega}^{N} f\left(\sigma_{q, \omega}^{k}(t)\right) \\
& =\frac{(1-q)\left(t-\omega_{0}\right)^{N-\alpha}}{\Gamma_{q}(N-\alpha)} \sum_{k=0}^{\infty} q^{k}\left(1-q^{k+1}\right)_{q, \omega}^{N-\alpha-1} D_{q, \omega}^{N} f\left(\sigma_{q, \omega}^{k}(t)\right) .
\end{aligned}
$$

In the following theorem, we introduce the properties of fractional Hahn difference operator of Caputo type.

Theorem 5.2 For $\alpha>0, q \in(0,1), \omega>0$, and $f: I_{q, \omega}^{T} \rightarrow \mathbb{R}$,

$$
{ }^{\mathrm{C}} D_{q, \omega}^{\alpha} \mathcal{I}_{q, \omega}^{\alpha} f(t)=f(t) .
$$

Proof For some $N-1<\alpha<N, N \in \mathbb{N}$, under Definition 5.1 and Theorem 4.3, we have

$$
\begin{aligned}
{ }^{C} D_{q, \omega}^{\alpha} \mathcal{I}_{q, \omega}^{\alpha} f(t) & =\mathcal{I}_{q, \omega}^{N-\alpha} D_{q, \omega}^{N} \mathcal{I}_{q, \omega}^{\alpha} f(t)=\mathcal{I}_{q, \omega}^{N-\alpha} D_{q, \omega}^{N-\alpha} f(t) \\
& =f(t)-\sum_{k=0}^{N-1} \frac{\left(t-\omega_{0}\right)^{k-\alpha}}{\Gamma_{q}(k-\alpha+1)}\left[D_{q, \omega}^{k} \mathcal{I}_{q, \omega}^{\alpha} f\left(\omega_{0}\right)\right] .
\end{aligned}
$$

From (3.1), we have

$$
\sum_{k=0}^{N-1} \frac{\left(t-\omega_{0}\right)^{k-\alpha}}{\Gamma_{q}(k-\alpha+1)}\left[D_{q, \omega}^{k} \mathcal{I}_{q, \omega}^{\alpha} f\left(\omega_{0}\right)\right]=0 .
$$

It implies that

$$
{ }^{\mathrm{C}} D_{q, \omega}^{\alpha} \mathcal{I}_{q, \omega}^{\alpha} f(t)=f(t)
$$

Theorem 5.3 For $\alpha>0, q \in(0,1), \omega>0$, and $f: I_{q, \omega}^{T} \rightarrow \mathbb{R}$,

$$
\mathcal{I}_{q, \omega}^{\alpha{ }^{C}} D_{q, \omega}^{\alpha} f(t)=f(t)-\sum_{k=0}^{N-1} \frac{\left(t-\omega_{0}\right)^{k}}{\Gamma_{q}(k+1)}\left[D_{q, \omega}^{k} f\left(\omega_{0}\right)\right],
$$

where $N-1<\alpha<N, N \in \mathbb{N}$.

Proof From Definition 5.1 and Theorem 5.1, we have

$$
\begin{aligned}
\mathcal{I}_{q, \omega}^{\alpha}{ }^{C} D_{q, \omega}^{\alpha} f(t) & =\mathcal{I}_{q, \omega}^{\alpha}\left[\mathcal{I}_{q, \omega}^{N-\alpha} D_{q, \omega}^{N} f(t)\right]=\mathcal{I}_{q, \omega}^{N} D_{q, \omega}^{N} f(t) \\
& =f(t)-\sum_{k=0}^{N-1} \frac{\left(t-\omega_{0}\right)^{k}}{\Gamma_{q}(k+1)}\left[D_{q, \omega}^{k} f\left(\omega_{0}\right)\right] .
\end{aligned}
$$


Corollary 5.1 Let $\alpha>0, q \in(0,1), \omega>0$, and $f: I_{q, \omega}^{T} \rightarrow \mathbb{R}$,

$$
\mathcal{I}_{q, \omega}^{\alpha{ }^{C}} D_{q, \omega}^{\alpha} f(t)=f(t)+C_{0}+C_{1}\left(t-\omega_{0}\right)+\cdots+C_{N-1}\left(t-\omega_{0}\right)^{N-1}
$$

for some $C_{i} \in \mathbb{R}, i=\mathbb{N}_{0, N-1}$, and $N-1<\alpha<N, N \in \mathbb{N}$.

\section{Conclusions}

In this paper, we have introduced a fractional Hahn integral, Riemann-Liouville and Caputo fractional Hahn difference operators. Many properties of these fractional Hahn operators have been proved. This work is certainly not complete and should be a starting point of many other works. For example, in future works, one could define the Laplace transform for Hahn calculus. Also, another work will be to find the Hahn-convolution product and compute its Hahn-Laplace transform, so we could be able to solve many more Hahn difference equations.

\section{Acknowledgements}

This research is supported by Suan Dusit University, Thailand.

Competing interests

The authors declare that they have no competing interests.

\section{Authors' contributions}

TS and TB worked together in the derivation of the mathematical results. All authors read and approved the final manuscript.

\section{Publisher's Note}

Springer Nature remains neutral with regard to jurisdictional claims in published maps and institutional affiliations.

Received: 10 August 2017 Accepted: 27 October 2017 Published online: 03 November 2017

\section{References}

1. Annaby, MH, Mansour, ZS: q-Fractional Calculus and Equations. Springer, Berlin (2012)

2. Kac, V, Cheung, P: Quantum Calculus. Springer, New York (2002)

3. Jagerman, DL: Difference Equations with Applications to Queues. Dekker, New York (2000)

4. Aldowah, KA, Malinowska, AB, Torres, DFM: The power quantum calculus and variational problems. Dyn. Contin. Discrete Impuls. Syst., Ser. B, Appl. Algorithms 19, 93-116 (2012)

5. Birto da Cruz, AMC, Martins, N, Torres, DFM: Symmetric differentiation on time scales. Appl. Math. Lett. 26(2), 264-269 (2013)

6. Cruz, B, Artur, MC: Symmetric quantum calculus. PhD thesis, Aveiro University (2012)

7. Wu, GC, Baleanu, D: New applications of the variational iteration method from differential equations to $q$-fractional difference equations. Adv. Differ. Equ. 2013, 21 (2013)

8. Tariboon, J, Ntouyas, SK: Quantum calculus on finite intervals and applications to impulsive difference equations. Adv. Differ. Equ. 2013, 282 (2013)

9. Álvarez-Nodarse, R: On characterization of classical polynomials. J. Comput. Appl. Math. 196, 320-337 (2006)

10. Hahn, W: Über Orthogonalpolynome, die q-Differenzenlgleichungen genügen. Math. Nachr. 2, 4-34 (1949)

11. Costas-Santos, RS, Marcellán, F: Second structure relation for q-semiclassical polynomials of the Hahn tableau. J. Math. Anal. Appl. 329, 206-228 (2007)

12. Kwon, KH, Lee, DW, Park, SB, Yoo, BH: Hahn class orthogonal polynomials. Kyungpook Math. J. 38, $259-281$ (1998)

13. Foupouagnigni, M: Laguerre-Hahn orthogonal polynomials with respect to the Hahn operator: fourth-order difference equation for the rth associated and the Laguerre-Freud equations recurrence coefficients. PhD thesis, Université Nationale du Bénin, Bénin (1998)

14. Aldwoah, KA: Generalized time scales and associated difference equations. PhD thesis, Cairo University (2009)

15. Annaby, MH, Hamza, AE, Aldwoah, KA: Hahn difference operator and associated Jackson-Nörlund integrals. J. Optim. Theory Appl. 154, 133-153 (2012)

16. Jackson, FH: Basic integration. Q. J. Math. 2, 1-16 (1951)

17. Malinowska, AB, Torres, DFM: The Hahn quantum variational calculus. J. Optim. Theory Appl. 147, 419-442 (2010)

18. Malinowska, AB, Torres, DFM: Quantum Variational Calculus. Springer Briefs in Electrical and Computer Engineering Control, Automation and Robotics. Springer, Berlin (2014)

19. Malinowska, $A B$, Martins, N: Generalized transversality conditions for the Hahn quantum variational calculus. Optimization 62(3), 323-344 (2013)

20. Hamza, AE, Ahmed, SM: Theory of linear Hahn difference equations. J. Adv. Math. 4(2), 441-461 (2013) 
21. Hamza, AE, Ahmed, SM: Existence and uniqueness of solutions of Hahn difference equations. Adv. Differ. Equ. 2013, 316 (2013)

22. Hamza, AE, Makharesh, SD: Leibniz's rule and Fubini's theorem associated with Hahn difference operator. J. Adv. Math. 12(6), 6335-6345 (2016)

23. Sitthiwirattham, $\mathrm{T}$ : On a nonlocal boundary value problem for nonlinear second-order Hahn difference equation with two different 9 , $\omega$-derivatives. Adv. Differ. Equ. 2016, 116 (2016)

24. Sriphanomwan, U, Tariboon, J, Patanarapeelert, N, Ntouyas, SK, Sitthiwirattham, T: Nonlocal boundary value problems for second-order nonlinear Hahn integro-difference equations with integral boundary conditions. Adv. Differ. Equ. $2017,170(2017)$

25. Čermák, J, Nechvátal, L: On (q, h)-analogue of fractional calculus. J. Nonlinear Math. Phys. 17(1), 51-68 (2010)

26. Čermák, J, Kisela, T, Nechvátal, L: Discrete Mittag-Leffler functions in linear fractional difference equations. Abstr. Appl. Anal. 2011, Article ID 565067 (2011)

27. Rahmat, MRS: The (q, h)-Laplace transform on discrete time scales. Comput. Math. Appl. 62, 272-281 (2011)

28. Rahmat, MRS: On some $(q, h)$-analogues of integral inequalities on discrete time scales. Comput. Math. Appl. 62 , 1790-1797 (2011)

29. Du, F, Jai, B, Erbe, L, Peterson, A: Monotonicity and convexity for nabla fractional $(q, h)$-difference. J. Differ. Equ. Appl. 22(9), 1224-1243 (2016)

\section{Submit your manuscript to a SpringerOpen ${ }^{\circ}$} journal and benefit from:

- Convenient online submission

- Rigorous peer review

- Open access: articles freely available online

- High visibility within the field

- Retaining the copyright to your article

Submit your next manuscript at $\boldsymbol{\nabla}$ springeropen.com 